PROCEEDINGS OF THE

AMERICAN MATHEMATICAL SOCIETY

Volume 133, Number 10, Pages 2817-2820

S 0002-9939(05)07905-0

Article electronically published on March 24, 2005

\title{
CENTRALIZER SIZES AND NILPOTENCY CLASS IN LIE ALGEBRAS AND FINITE $p$-GROUPS
}

\author{
A. JAIKIN-ZAPIRAIN
}

(Communicated by Jonathan I. Hall)

\begin{abstract}
In this work we solve a conjecture of Y. Barnea and M. Isaacs about centralizer sizes and the nilpotency class in nilpotent finite-dimensional Lie algebras and finite $p$-groups.
\end{abstract}

\section{INTRODUCTION}

By a well-known result of N. Ito [3], a finite group with only two conjugacy class sizes (one of which must be 1) is nilpotent. Recently K. Ishikawa 2] showed that the nilpotency class of such a group is at most 3. This result was an open question for many years. A simplification of Ishikawa's argument was given by M. Isaacs, and his proof is presented in [1. Note that the number of different conjugacy class sizes in a finite group $G$ is equal to the number of different orders of centralizers of elements of $G$. This observation allows us to consider possible analogs of Ito's and Ishikawa's theorems for Lie algebras: What can be said about a finite-dimensional Lie algebra $L$ if the centralizer subalgebras $C_{L}(x)$ have just two different dimensions as $x$ runs over the elements of $L$ ? This question was considered by Y. Barnea and M. Isaacs in [1. In particular, when $L$ is nilpotent they proved that, as in Ishikawa's Theorem, the nilpotency class of $L$ is at most 3 .

If $L$ is a finite-dimensional non-abelian Lie algebra, then denote by $\operatorname{cd}(L)$ the set of dimensions of centralizers of noncentral elements of $L$. Denote by $\Delta(L)$ the difference $\max (\operatorname{cd}(L))-\min (\operatorname{cd}(L))$. Similarly, if $G$ is a non-abelian finite $p$-group, denote by $\operatorname{cs}(G)$ the set of orders of centralizers of noncentral elements of $G$ and let $\Delta(G)$ be the difference $\log _{p} \max (\operatorname{cs}(G))-\log _{p} \min (\operatorname{cs}(G))$. Thus, $\Delta(G)=0$ corresponds exactly to the assumption in Ishikawa's theorem. This observation led Barnea and Isaacs to conjecture the following generalization of Ishikawa's result:

Conjecture ([1, Conjectures E and F]).

(1) Let $L$ be a nilpotent Lie algebra. Then the nilpotence class of $L$ is bounded in terms of $\Delta(L)$.

(2) Let $G$ be a finite p-group. Then the nilpotency class of $G$ is bounded in terms of $\Delta(G)$.

Received by the editors May 19, 2004.

2000 Mathematics Subject Classification. Primary 20D15; Secondary 17B30.

Key words and phrases. p-groups, nilpotent Lie algebras, conjugacy class sizes.

This work was partially supported by the MCYT Grants BFM2001-0201, BFM2001-0180, FEDER and the Ramón y Cajal Program. 
In this paper we prove this conjecture. In the following we will write $\left[N,{ }_{k} M\right]$ for $[N, M, \ldots, M]=[\ldots[N, M], \ldots, M]$, where $M$ appears $k$ times. If $L$ is a nilpotent Lie algebra we denote by $L^{i}$ the $i$ th term of the lower central series (i.e. $L^{i}=$ $\left.\left[L,{ }_{i-1} L\right]\right)$. We denote by $\operatorname{cl}(L)$ the nilpotency class of $L$. We use a similar notation for $p$-groups: $G^{i}$ denotes the $i$ th term of the lower central series of a $p$-group $G$ and $\operatorname{cl}(G)$ denotes the nilpotency class of $G$. Now we are ready to state our results.

Theorem 1.1. Let $L$ be a nilpotent finite-dimensional Lie algebra. Then

$$
L^{\Delta(L)+3} \subseteq Z(L)+L^{\prime \prime} \text { and } \operatorname{dim}\left(L^{\prime \prime}+Z(L) / Z(L)\right) \leq \Delta(L)(2 \Delta(L)+1) .
$$

In particular, $\operatorname{cl}(L) \leq 2 \Delta(L)^{2}+2 \Delta(L)+3$.

Theorem 1.2. Let $G$ be a finite p-group. Then

$$
G^{\Delta(G)+3} \subseteq Z(G) G^{\prime \prime} \text { and }\left|G^{\prime \prime} Z(G) / Z(G)\right| \leq p^{\Delta(G)(2 \Delta(G)+1)} .
$$

In particular, $\operatorname{cl}(G) \leq 2 \Delta(G)^{2}+2 \Delta(G)+3$.

As a consequence of Theorem 1.1 and $[1$, Theorems $\mathrm{C}$ and $\mathrm{D}]$ we obtain the following corollary.

Corollary 1.3. Let $L$ be a finite-dimensional complex Lie algebra. Then L contains a nilpotent ideal $N$ such that $\operatorname{dim}(L / N)$ and the nilpotency class of $N$ are bounded in terms of $\Delta(L)$.

Finally we would like to pose two further questions for future investigations.

Conjecture A. Corollary 1.3 is stil true when $L$ is a Lie algebra over an arbitrary field.

Conjecture B. Let $L$ be a nilpotent Lie algebra. Then the derived length of $L$ is bounded in terms of $|\mathrm{cd}(L)|$.

\section{Proofs of the main Results}

Proof of Theorem 1.1. Let $m$ be the nilpotency class of $L$ (so that $L^{m} \neq 0$ and $L^{m+1}=0$ ) and assume that $m>3$ (otherwise the theorem is trivial). We have $\left[L^{m-2}, L, L\right]=L^{m}>0$, and thus $\left[L^{m-2}, L\right] \nsubseteq Z Z(L)$. Since the set $\left\{r \in L^{m-2}\right.$ $[r, L] \subseteq Z(L)\}$ is a proper subalgebra of $L^{m-2}$, there exists an element $u \in L^{m-2}$ that does not lie in this set. Then the set $R=\{t \in L \mid[u, t] \in Z(L)\}$ is a proper subalgebra of $L$. Choose $v \in L \backslash R$ and write $x=[u, v]$, so that $x \in L^{m-1}$. Recall that $\left[L^{i}, L^{j}\right] \subseteq L^{i+j}$ for all superscripts $i, j \geq 1$, and thus $\left[L^{2}, v, u\right] \subseteq\left[L^{3}, L^{m-2}\right] \subseteq$ $L^{m+1}=0$.

Let $S=[L, v, u]$ and $T=\left[L^{m-1}, v\right]$. We have

$$
[L, x]=[L,[u, v]] \subseteq[L, v, u]+[L, u, v] \subseteq S+\left[L^{m-1}, v\right]=S+T
$$

and thus

$$
\operatorname{dim}(S)+\operatorname{dim}(T) \geq \operatorname{dim}([L, x])=\operatorname{dim}(L)-\operatorname{dim}\left(C_{L}(x)\right) .
$$

Notice that $L^{\prime}+C_{L}(v) \subseteq \operatorname{ker}(\operatorname{ad} u \circ$ ad $v)$. Hence

$$
\operatorname{dim} S \leq \operatorname{dim} L-\operatorname{dim}\left(L^{\prime}+C_{L}(v)\right) .
$$

Thus, using (2.1), we obtain that

$$
\operatorname{dim} L-\operatorname{dim}\left(L^{\prime}+C_{L}(v)\right)+\operatorname{dim} L^{m-1}-\operatorname{dim} C_{L^{m-1}}(v) \geq \operatorname{dim}(L)-\operatorname{dim}\left(C_{L}(x)\right) .
$$


Since $\operatorname{dim} L^{m-1}-\operatorname{dim} C_{L^{m-1}}(v)=\operatorname{dim}\left(C_{L}(v)+L^{m-1}\right)-\operatorname{dim} C_{L}(v)$, we have

$$
\operatorname{dim}\left(L^{\prime}+C_{L}(v)\right)-\operatorname{dim}\left(C_{L}(v)+L^{m-1}\right) \leq \operatorname{dim}\left(C_{L}(x)\right)-\operatorname{dim}\left(C_{L}(v)\right) .
$$

Therefore, we obtain that

$$
\begin{aligned}
\operatorname{dim} L^{\prime}-\operatorname{dim}\left(\left(C_{L}(v)+L^{m-1}\right) \cap L^{\prime}\right) & =\operatorname{dim}\left(L^{\prime}+C_{L}(v)\right)-\operatorname{dim}\left(C_{L}(v)+L^{m-1}\right) \\
& \leq \operatorname{dim}\left(C_{L}(x)\right)-\operatorname{dim}\left(C_{L}(v)\right) \leq \Delta(L) .
\end{aligned}
$$

Hence, if we denote $L / L^{m}$ by $\bar{L}$, we obtain that for any $\bar{v} \in \bar{L} \backslash \bar{R}$,

$$
\operatorname{dim}\left(\bar{L}^{\prime} / C_{\bar{L}^{\prime}}(\bar{v})\right) \leq \Delta(L) .
$$

If $y \in \bar{L} \backslash \bar{R}$ and $z \in \bar{L}^{\prime}$, then $y+z \in \bar{L} \backslash \bar{R}$. Thus, we obtain that

$$
\operatorname{dim}\left(\bar{L}^{\prime} / C_{\bar{L}^{\prime}}(z)\right) \leq \operatorname{dim}\left(\bar{L}^{\prime} /\left(C_{\bar{L}^{\prime}}(y) \cap C_{\bar{L}^{\prime}}(z+y)\right)\right) \leq 2 \Delta(L) .
$$

By [4], $\operatorname{dim} \bar{L}^{\prime \prime} \leq \Delta(L)(2 \Delta(L)+1)$.

Now, let $\hat{L}=L /\left(L^{\prime \prime}+Z(L)\right)$ and put $D=\hat{L} \backslash \hat{R}$. Then $D$ spans $\hat{L}$. If $y \in D$, then $\left[\hat{L}^{\prime}, y\right]$ is an ideal of $\hat{L}$ because the derived length of $\hat{L}$ is 2 . On the other hand, we proved that $\operatorname{dim}\left[\hat{L}^{\prime}, y\right] \leq \Delta(L)$. Since $\hat{L}$ is nilpotent, $\left[\hat{L}^{\prime}, y, \Delta(L) \hat{L}\right]=0$ and so $\hat{L}^{\Delta(L)+3}=0$.

Proof of Theorem 1.2. Let $G=G^{1}>G^{2}>\cdots>G^{m}>G^{m+1}=1$ be the lower central series of $G$, where $m$ is the nilpotency class, and assume that $m>3$ (otherwise the theorem is trivial). We have $\left[G^{m-2}, G, G\right]=G^{m}>\{1\}$, and thus $\left[G^{m-2}, G\right] \not Z Z(G)$. Since the set $\left\{r \in G^{m-2} \mid[r, G] \subseteq Z(G)\right\}$ is a proper subgroup of $G^{m-2}$, there exists an element $u \in G^{m-2}$ that does not lie in this set. Then the set $R=\{t \in G \mid[u, t] \in Z(G)\}$ is a proper subgroup of $G$. Choose $v \in G \backslash R$ and write $x=[u, v]$, so that $x \in G^{m-1}$. Recall that $\left[G^{i}, G^{j}\right] \subseteq G^{i+j}$ for all superscripts $i, j \geq 1$, and thus $\left[G^{2}, v, u\right] \subseteq\left[G^{3}, G^{m-2}\right] \subseteq G^{m+1}=1$.

Next, we define the map $W: G \rightarrow G$ by means of $W(g)=[g, v, u]$ for all $g \in G$. Note that $\left[x_{1}, x_{2}, x_{3}, x_{4}\right]=1$ if some $x_{i}$ is $u$ and some $x_{j}$ is $v$. Hence for any $g, h \in G$,

$$
W(g h)=[g h, v, u]=[[g, v][g, v, h][h, v], u]=[g, v, u][h, v, u]=W(g) W(h),
$$

and so $W$ is a homomorphism of groups.

Now let $y \in G$ be arbitrary. We have $u \in G^{m-2}$ and, of course, $v, y \in G^{1}$, and so the Witt identity applies and we have $[v, u, y][y, v, u][u, y, v]=1$. Since $[u, v]=x$, it follows that

$$
[y, v, u][u, y, v]=[y,[v, u]]=[x, y] .
$$

Hence $\left|G: \operatorname{ker} W \| G^{m-1}: C_{G^{m-1}}(v)\right| \geq\left|G: C_{G}(x)\right|$. Since $G^{\prime} C_{G}(v) \leq \operatorname{ker} W$ we obtain that

and so

$$
\frac{|G|\left|G^{m-1} C_{G}(v)\right|}{\left|G^{\prime} C_{G}(v)\right|\left|C_{G}(v)\right|} \geq \frac{|G|}{\left|C_{G}(x)\right|}
$$

$$
\frac{\left|G^{\prime}\right|}{\left|\left(G^{m-1} C_{G}(v)\right) \cap G^{\prime}\right|}=\frac{\left|G^{\prime} C_{G}(v)\right|}{\left|G^{m-1} C_{G}(v)\right|} \leq \frac{\left|C_{G}(x)\right|}{\left|C_{G}(v)\right|} \leq p^{\Delta(G)} .
$$

Denote $G / G^{m}$ by $\bar{G}$. Then from the last inequality we obtain that

$$
\left|\bar{G}^{\prime}: C_{\bar{G}^{\prime}}(\bar{v})\right| \leq p^{\Delta(G)}
$$


If $y \in \bar{G} \backslash \bar{R}$ and $z \in \bar{G}^{\prime}$, then $y z \in \bar{G} \backslash \bar{R}$. Thus, we obtain that

$$
\left|\bar{G}^{\prime}: C_{\bar{G}^{\prime}}(z)\right| \leq\left|\bar{G}^{\prime}:\left(C_{\bar{G}^{\prime}}(y) \cap C_{\bar{G}^{\prime}}(z y)\right)\right| \leq p^{2 \Delta(G)} .
$$

By $\left[4,\left|\bar{G}^{\prime \prime}\right| \leq p^{\Delta(G)(2 \Delta(G)+1)}\right.$.

Now, let $\hat{G}=G /\left(G^{\prime \prime} Z(G)\right)$ and put $D=\hat{G} \backslash \hat{R}$. Then $D$ generates $\hat{G}$. If $y \in D$, then $\left[\hat{G}^{\prime}, y\right]$ is a normal subgroup of $\hat{G}$ because the derived length of $\hat{G}$ is 2 . On the other hand, by (2.2), $\left|\left[\hat{G}^{\prime}, y\right]\right| \leq p^{\Delta(G)}$. Since $\hat{G}$ is nilpotent, $\left[\hat{G}^{\prime}, y, \Delta(G) \hat{G}\right]=1$ and so $\hat{G}^{\Delta(G)+3}=1$.

\section{ACKNOWLEDGMENTS}

This work was done during my visit to the Imperial College of London and I thank the Department of Mathematics and personally M. Liebeck for their hospitality. I also thank Y. Barnea for some helpful comments on a preliminary version of this work.

\section{REFERENCES}

[1] Y. Barnea and I. M. Isaacs, Lie algebras with few centralizer dimensions, J. Algebra 259 (2003), 284-299. MR1953720 (2004a:17005)

[2] K. Ishikawa, On finite p-groups which have only two conjugacy lengths, Israel J. Math. 129 (2002), 119-123. MR1910937 (2004b:20032)

[3] N. Ito, On finite groups with given conjugate types. I, Nagoya Math. J. 6 (1953), 17-28. MR:0061597(15:851c)

[4] M. R. Vaughan-Lee, Breadth and commutator subgroups of p-groups, J. Algebra 32 (1974), 278-285. MR0364436(51:690)

Departamento de Matemáticas, Facultad de Ciencias, Universidad Autónoma de MADRID, 28049 MADRID, SPAIN

E-mail address: andrei.jaikin@uam.es 